\title{
OUTCOME OF ATRIAL FIBRILLATION AFTER MITRAL VALVE REPAIR
}

Jean F. Obadia, MD, PhD

Mazen El Farra, MD

Olivier H. Bastien, MD

Michel Lièvre, MD

Yvan Martelloni, MD

Jean F. Chassignolle, MD
Objective: The aim of the study was to evaluate the prognostic factors for return to sinus rhythm after mitral valve repair. Method: One hundred ninety-one patients underwent surgery for mitral valve repair, including 142 procedures for valve repair only $(74 \%)$. The patients with preoperative atrial fibrillation (50.5\%) were older, clinically more symptomatic, and had a greater degree of left atrial dilation than the patients who had sinus rhythm. Results: Preoperative cardiac rhythm, the duration of preoperative atrial fibrillation, and a lesser degree of left atrial hypertrophy are significant prognostic factors independent of the maintenance of sinus rhythm. The probability of return to stable sinus rhythm was $93.7 \%$ when sinus rhythm was already present before the operation and $80 \%$ when atrial fibrillation was intermittent or of less than 1 year's duration; probability declined abruptly for durations over 1 year. No significant difference in patient survival was noted between those who had sinus rhythm $(99 \% \pm$ $0.9 \%$ at 1 year and $86 \% \pm 6.6 \%$ at 5 years) and those who had atrial fibrillation in the preoperative period $(95 \% \pm 3.1 \%$ at 1 year and $86 \% \pm$ $8.4 \%$ at 5 years). In contrast, the postoperative return to sinus rhythm was associated with $99 \% \pm 0.9 \%$ and $94 \% \pm 4.8 \%$ survivals at 1 and 4 years versus $97 \% \pm 1.5 \%$ and $77 \% \pm 13 \%$ in the event of postoperative atrial fibrillation. Conclusion: The aim of restoring postoperative sinus rhythm after mitral valve repair should lead to surgery being conducted on patients who have sinus rhythm or recent-onset atrial fibrillation. Surgery for atrial fibrillation may be of value in patients with a long history of atrial fibrillation, providing that it does not induce prohibitive excess mortality. (J Thorac Cardiovasc Surg 1997;114:179-85)
$T^{1}$ he surgical repair of mitral incompetence affords numerous advantages such as the hemodynamic effects related to conservation of the subvalvular apparatus, ${ }^{1,2}$ but the most attractive feature is indubitably the prospect of maintaining or restoring sinus rhythm (SR). In addition to increasing cardiac output, restoration of SR avoids long-term oral anticoagulant treatment. During the study, we endeavored to determine the prognostic factors for SR restoration in patients undergoing operations for mitral valve repair. These factors should also con-

From the Hôpital Cardiologique Louis Pradel, Lyon, France.

Received for publication Dec. 16, 1996; revisions requested Feb. 14, 1997; revisions received March 11, 1997; accepted for publication March 13, 1997.

Address for reprints: Jean F. Obadia, MD, PhD, Service de Chirurgie Cardio-Thoracique, Hôpital Cardiologique, Boulevard Pinel, 69003 Lyon, France.

Copyright $(1997$ by Mosby-Year Book, Inc.

$0022-5223 / 97 \$ 5.00+0 \quad \mathbf{1 2 / 1 / 8 1 9 4 1}$ tribute to defining the potential role of surgery in atrial fibrillation (AF) in association with mitral repair. $^{3}$

\section{Patients and methods}

Patients. From November 11, 1991, to March 3, 1996, a series of 191 consecutive patients (124 men and 67 women) aged $64 \pm 12$ years (range 30 to 86 years) underwent operations for mitral valve repair. Dyspnea of New York Heart Association (NYHA) class III or IV was present in $55 \%$ of the patients and angina pectoris in $19 \%$. Mitral regurgitation was mostly severe (grades III and IV), with only six patients in grade II having combined operations (three coronary bypasses and three aortic valvular replacements). In 142 patients (74\%) the surgical procedure consisted in mitral valve repair only. In other patients, combined operations were involved: 33 coronary bypasses $(17.3 \%), 6$ aortic valve replacements $(3.1 \%), 2$ aortic valve repairs $(1 \%), 2$ left ventricular aneurysm corrections (1\%), and 7 tricuspid valve repairs (3.6\%). Three patients $(1.6 \%)$ had previously undergone coronary bypass grafting. The mitral lesions consisted in 156 cases of dystrophy (81.2\%), 17 cases of ischemic lesions (8.9\%), 11 cases of endocarditis $(5.7 \%)$, of which 6 necessitated 
Table I. Probability of SR resuming after mitral valve repair as a function of preoperative rhythm and $A F$ duration

\begin{tabular}{lcccc}
\hline & $\begin{array}{c}\text { Preoperative rhythm in } \\
\text { 191 patients undergoing } \\
\text { mitral valve repair }\end{array}$ & No. & $\begin{array}{c}\text { No. of patients with } \\
\text { postoperative SR }\end{array}$ & $\begin{array}{c}\text { Percentage of patients with } \\
\text { retum to SR }\end{array}$ \\
\cline { 2 - 5 } & $\%$ & 95 & 89 & 93.7 \\
SR & 50 & 29 & 23 & 79.3 \\
Intermittent AF & 15 & 20 & 17 & 85 \\
$\mathrm{AF}<3$ mo & 11 & 12 & 8 & 66.7 \\
$\mathrm{AF} 3$ mo to 1 yr & 6 & 14 & 5 & 35.7 \\
$\mathrm{AF} 1$ to $3 \mathrm{yr}$ & 7 & 21 & 1 & 4.5 \\
$\mathrm{AF}$ over $3 \mathrm{yr}$ & 11 & & & \\
\hline
\end{tabular}

$S R$, Sinus rhythm; $A F$, atrial fibrillation.

Table II. Clinical characteristics of 191 patients operated on for mitral valvuloplasty as a function or preoperative atrial rhythm

\begin{tabular}{lccc}
\hline Preoperative cardiac rhythm & $\begin{array}{c}S R \\
(n=95)\end{array}$ & $\begin{array}{c}A F \\
(n=96)\end{array}$ & $p$ Value \\
\hline Mean age (yr) & $62.1 \pm 14$ & $66 \pm 9$ & $p=0.04$ \\
Sex (\% male) & 70.3 & 61.4 & $p=0.50$ \\
NYHA class I and II (\%) & 56.2 & 41.8 & $p=0.03$ \\
Mean left atrial diameter (mm) & $50.25 \pm 10$ & $55.36 \pm 9$ & $p=0.01$ \\
Mean TDV (mm) & $56.67 \pm 8$ & $60.67 \pm 9$ & $p=0.30$ \\
Mean EF (\%) & $57.5 \pm 13$ & $55 \pm 9.15$ & $p=0.50$
\end{tabular}

$S R$, Patients with preoperative sinus rhythm; $A F$, patients with permanent or intermittent preoperative atrial fibrillation; $N Y H A$, New York Heart Association; $T D V$, tele-diastolic volume; $E F$, ejection fraction.

surgery in the acute phase, 6 cases of rheumatic lesions $(3.1 \%)$, and 2 cases of congenital lesions $(1 \%)$.

Before the operation, SR was present in 95 patients (49.5\%) and $\mathrm{AF}$ in $96(50.5 \%)$. AF was permanent in 68 patients $(35.4 \%)$, permanent being defined as no return at any time to SR. The durations of $\mathrm{AF}$ are summarized in Table I. Twenty-nine patients (15.1\%) had intermittent episodes of AF. AF was considered to be intermittent when episodes of $\mathrm{AF}$ and $\mathrm{SR}$ were observed in a patient even if SR was present at the time of the operation. The latter group was considered separately because we postulated that the patients having had several, even brief, episodes of $\mathrm{AF}$ reduced by pharmacologic or electrical cardioversion over the months or years preceding the operation might react differently from the two other patient groups. The history of intermittent AF was variable and ranged from 2 months to 12 years (mean $=11.6$ months). Two patients with SR and two patients with AF had had pacemaker implantations.

Comparison of patients with and without AF. The clinical and hemodynamic data are summarized in Table II. The patients with permanent or intermittent preoperative AF were older, more symptomatic, in a more pejorative mean NYHA class, and had a greater degree of left atrial dilation according to ultrasonography. The proportion of women seemed higher, but this difference did not reach statistical significance. No significant differences were observed in hemodynamic parameters such as end-diastolic volume and ejection fraction determined by ultrasonography. We observed more episodes of throm- boembolic complications among the patients who had preoperative intermittent $\mathrm{AF}$ or permanent $\mathrm{AF}$ (five versus none in the $\mathrm{SR}$ group, $p=0.04)$. Among the 33 patients with associated coronary bypass operations, 45.5\% had SR and 50.5\% had AF. This difference was not statistically significant.

Surgical procedure. The operations were performed with the use of standard cardiopulmonary bypass with normothermia and antegrade cold blood cardioplegia. The approach consisted in sternotomy for 190 patients and right thoracotomy for two young women. The mitral valve was approached through a standard left atriotomy made just posterior to the interatrial groove in 174 patients or by a transseptal approach in 10 patients. The latter route was reserved for repeat operations or small left atria for which complex repair was scheduled. Repair involved the posterior leaflet 157 times (81.8\%), the anterior leaflet 11 times $(5.7 \%)$, and both leaflets 24 times $(12.5 \%)$. The surgical techniques consisted in the following alone or in combination: 182 quadrangular resections, 99 chordal transpositions, 18 chordal shortenings, and 10 major decalcifications of the posterior leaflet. Annuloplasty was effected to reduce annular dimension and protect leaflet repair in all patients but three $(98.4 \%)$. In the majority of patients (93.8\%), a Physioring or rigid Carpentier ring (Baxter Healthcare Corporation, Edwards CVS Division, Irvine, Calif.) was used for annuloplasty. In the other patients, a Duran ring was used. In all patients, the left atrial appendage was excluded by endocardiac suture. 
Anticoagulant management. Continuous intravenous anticoagulant treatment with heparin was initiated immediately after the operation. On day 5, medication was switched to oral anticoagulants. The required INR (international normalized ratio) was between 3 and 4.5. No patients received platelet aggregation inhibitors. The oral anticoagulants were maintained for at least 3 months if the patient had SR in the postoperative period or for at least 2 months after effective cardioversion, if restoration of SR was obtained secondarily.

Data collection and follow-up. Prospective computerized data entry was performed in the authors' department for all patients. All surviving patients either were seen again at consultations or were mailed a questionnaire. The data entered in the database were completed or confirmed by a survey of attending physicians. The cumulative follow-up was 458.4 years (mean follow-up $2.4 \pm 1.1$ years). No patient was lost to follow-up in the series. Follow-up was therefore of relatively short duration, but exhaustive.

Statistical analysis. The definitions used are those recommended by the Society of Thoracic Surgeons and The American Association for Thoracic Surgery. ${ }^{4}$ Frequencies were compared by means of the $\chi^{2}$ test with Yates' correction. Actuarial survival was calculated by means of the Kaplan-Meier method. ${ }^{5}$ The log rank test was used to test for statistically significant observed differences. All data are reported as mean \pm standard deviations.

The role of left atrial diameter and preoperative AF duration in predicting the return of SR was studied by means of logistic regression. Because the duration of $\mathrm{AF}$ obeyed a non-Gaussian distribution, the $\log$ of duration (expressed in months) was used. Testing for correlation between AF duration and atrial diameter was also conducted. The statistical analysis was conducted with the use of the STATISTICA Software for Windows package (StatSoft, Inc., Tulsa, Okla.).

\section{Results}

Postoperative mortality and morbidity. The overall 30-day mortality rate was $4.7 \%$ (nine patients). The one death in the 86 patients with NYHA class I or II disease $(1.2 \%)$ was due to a postoperative toxic/allergic reaction with generalized peripheral myolysis and anuria. Mortality in the 96 patients with nonurgent class III and IV disease was $3.1 \%$ (three patients including two with concomitant coronary revascularizations). Mortality was higher in the subgroup requiring emergency procedures, in which four of 10 patients died. The early mortality (in 182 patients with nonurgent treatment) was similar for patients with preoperative SR and for those with permanent or intermittent preoperative $\mathrm{AF}$.

As evidenced by the prevalence of inotropic support during weaning from bypass $(22 \%$ of patients with preoperative SR and $43 \%$ of patients with permanent or intermittent $\mathrm{AF}, p=0.032$ ) and intraaortic balloon use $(1.9 \%$ of patients with preoperative SR and $3.9 \%$ of patients with permanent or intermittent AF, $p=0.30$ ), the occurrence of low postoperative cardiac outputs seemed less marked in the patients with preoperative SR.

No significant difference was noted between groups in major postoperative complications including all degrees of neurologic complications.

Repeat operations. Two early valve replacements $(1 \%)$ were required during the first postoperative month, because of defective initial repair. One patient underwent a second operation on day 12 to resecure a Carpentier mitral ring that had dehisced as a result of a technical error. All three patients had uncomplicated courses thereafter. Four patients underwent second operations unrelated to the quality of the initial repair: Two patients had acute endocarditis with early recurrence of vegetations necessitating reoperation and prosthetic valve replacement 4 and 5 weeks after repair; one patient had heart transplantation and another had secondary infectious endocarditis occurring 2 and 3.5 years after repair. No secondary valve replacement was necessary.

Survival. The overall proportion of patients surviving in the series was $94 \% \pm 2.9 \%$ at 1 year and $84 \% \pm 9.2 \%$ at 5 years. No significant difference (Fig. 1) was noted between the survival of patients who had preoperative SR $(99 \% \pm 0.9 \%$ at 1 year and $86 \% \pm 6.6 \%$ at 5 years) and those who had permanent or intermittent preoperative AF $95 \% \pm$ $3.1 \%$ at 1 year and $86 \% \pm 8.4 \%$ at 5 years). Nevertheless, the return to stable postoperative SR in patients having survived surgery yielded actuarial survivals of $99 \% \pm 0.9 \%$ at 1 year and $92 \% \pm 5.6 \%$ at 5 years versus $97 \% \pm 1.5 \%$ at 1 year and $77 \% \pm$ $13 \%$ at 4 years for the patients who had postoperative AF (Fig. 2). This difference did not, however, reach the significance level in the log rank test because of the small size of the postoperative AF subgroup $(n=41)$. This also explains why survival is given only to 4 years in this subgroup.

Thromboembolic events. Two cases of transient neurologic complications were observed after the operation. No secondary thromboembolic event was observed in the patients with postoperative SR. Two thromboembolic complications were observed in those with postoperative AF. The difference was not statistically significant.

Clinical results and restoration of SR. The clinical and hemodynamic results are summarized in Table III. Only $31 \%$ of the patients had SR when they were weaned from extracorporeal circulation 


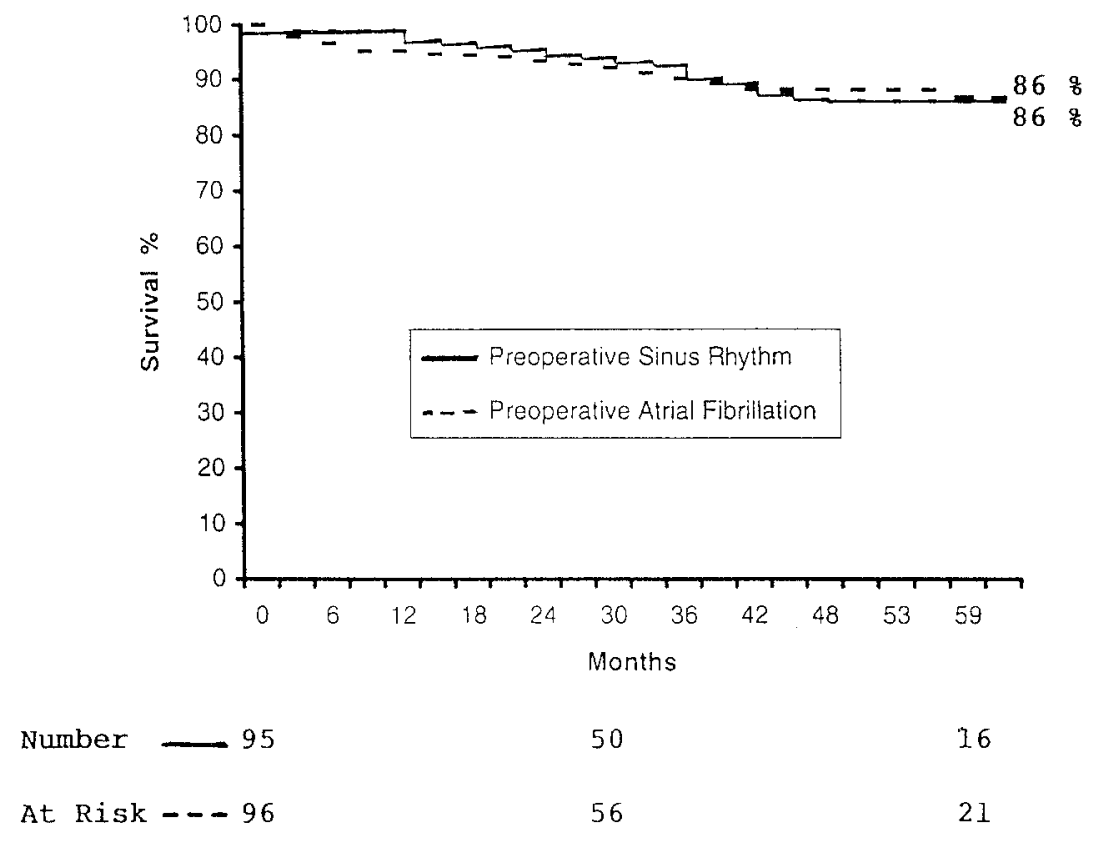

Fig. 1. Actuarial survival as a function of preoperative cardiac rhythm: 95 patients with SR and 96 patients with permanent or intermittent AF.

and remained in SR subsequently. In the other patients, AF was present at the time of weaning from extracorporeal circulation (38\%) or AF developed during the postoperative period (31\%). Among the patients who had secondary recovery of stable SR, resumption of SR was spontaneous in $20 \%$, was obtained with amiodarone in $66 \%$, or resulted from electrical cardioversion in $14 \%$. Among the patients with stable postoperative SR, those who had SR in the preoperative period were less likely to have intermittent $\mathrm{AF}$ in the postoperative period (58\% vs $78 \%, p=0.38$ ), and SR resumed earlier, during the first few months ( $75 \%$ vs $52 \%, p=0.29$ ), than in the other patients.

Overall, $49.5 \%$ of patients had preoperative SR compared with $74.9 \%$ at the late follow-up time point. The probability of return to stable SR (see Table I) was $93.7 \%$ when SR was present before the operation and $80 \%$ when AF was intermittent or of a duration less than 1 year. In contrast, in patients with AF lasting beyond 1 year, the probability of return to SR fell abruptly.

Among the 143 patients with stable postoperative SR, $68 \%$ required no antiarrhythmic drugs to maintain SR.

Return to stable SR, as evidenced in the late follow-up period, also depended on the preoperative dimensions of the left atrium. The mean preoperative diameter was $50.25 \mathrm{~mm}$ in the patients in whom stable postoperative SR returned versus $56.1 \mathrm{~mm}$ in those who continued to have $\mathrm{AF}(p=0.006)$.

Logistic regression analysis of patients with preoperative AF (Fig. 3). Left atrial diameter and the logarithm of AF duration were significantly correlated $\left(r=0.58, p<10^{-6}\right)$. Both were significant and independent predictive factors of the return to SR ( $p=0.032$ and $p=0.0006$, respectively). The model yielded a correct prediction of AF persistence in $85 \%$ of patients and a correct prediction of return to SR in $88 \%$ of patients.

Age, sex, the cause of the mitral valve disease, and the occurrence of an associated procedure did not correlate with the presence of SR at late follow-up.

Regression of atrial and ventricular diameter. Presence of SR favorably influenced a decrease in left atrial size (from $50.12 \mathrm{~mm}$ before the operation to $42.16 \mathrm{~mm}$ after the operation) compared with persistent AF (from $54.81 \mathrm{~mm}$ to $50.62 \mathrm{~mm} ; p=$ $0.03)$. Conversely, return to SR did not influence the regression of the ventricular size.

\section{Comments}

The negative effects of AF are widely known, particularly when $\mathrm{AF}$ is accompanied by mitral valve disease. ${ }^{6,7}$ The aim of the present study was there- 


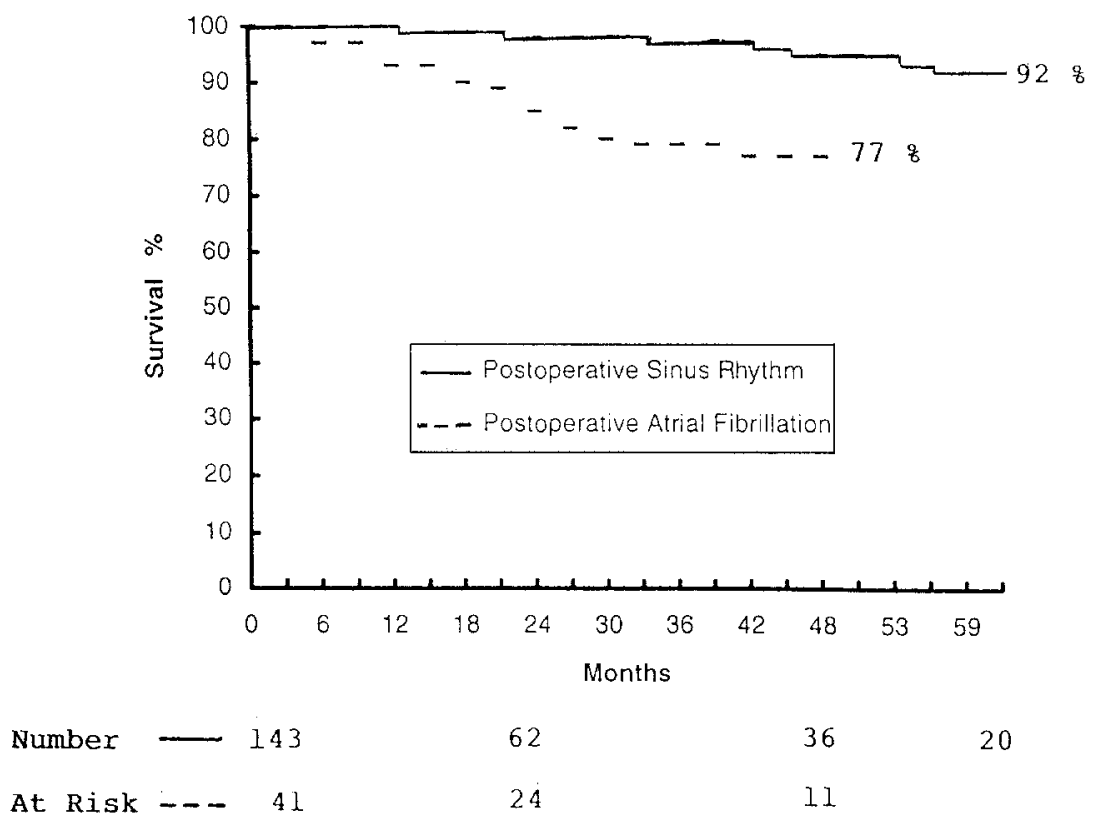

Fig. 2. Actuarial survival as a function of postoperative cardiac rhythm: 144 patients with SR and 41 patients with permanent or intermittent AF. Postoperative deaths were excluded.

fore to determine the prognostic factors with respect to SR maintenance after mitral valve repair.

The most positive prognostic factor was the presence of preoperative SR. SR was maintained after the operation in more than $90 \%$ of patients. In the present series, about half the patients with preoperative AF had stable SR in the postoperative period. In patients with a history of intermittent AF, even of long duration, or with recent-onset $\mathrm{AF}$, the probability of a return to SR remains good. However, the risk of $\mathrm{AF}$ persisting after valve repair becomes major in the presence of chronic preoperative AF lasting more than 1 year. In addition, SR recovery occurs early, with fewer episodes of $\mathrm{AF}$ during the postoperative period, in patients having preoperative SR. This may therefore promote earlier functional recovery. However, this recovery is not immediate if the patient has a history of AF. Several weeks are sometimes required for the left atrium to become smaller ${ }^{8}$ and atrial contractile function to reemerge. ${ }^{9}$

The second prognostic factor, independent of the preceding one in multivariate analysis, is the preoperative diameter of the left atrium. The probability of a return to SR is low when the left atrial diameter is greater than $60 \mathrm{~mm} .{ }^{10}$ However, the predictive value of preoperative left atrial diameter remains
Table III. Clinical and hemodynamic results after mitral valve repair

\begin{tabular}{lccc}
\hline & Preoperative & Postoperative & $p$ Value \\
\hline NYHA class & & & \\
I-II & 87 & $171^{*}$ & $p<0.00001$ \\
III-IV & 105 & $7^{*}$ & \\
TDV & $62.66 \pm 8.66$ & $52.78 \pm 6.6^{*}$ & $p \times 0.001$ \\
EF & $58.99 \pm 14.75$ & $54.14 \pm 15.2^{*}$ & $p \times 0.04$ \\
LA & $52.84 \pm 9.81$ & $44.86 \pm 9.15^{*}$ & $p \times 0.01$
\end{tabular}

$N Y H A$, New York Heart Association; $T D V$, tele-diastolic volume; $E F$, ejection fraction; $L A$, echographic left atrial size.

"Postoperative deaths excluded.

lower than that of preoperative cardiac rhythm or duration of $\mathrm{AF}$. The postoperative evolution of the atrial size was clearly different in the patients in whom SR resumed, but we cannot say whether it is the decreased size of the atrium that allows the return to SR or whether it is the SR that permits a better reduction of the atrial size.

No other prognostic factors for return to SR were identified. In addition, the two significant criteria, preoperative rhythm and left atrial diameter, may prove to be inadequate predictors, particularly in borderline cases: stable SR resumed in $36 \%$ of the patients who had had AF for 1 to 3 years and $22 \%$ 


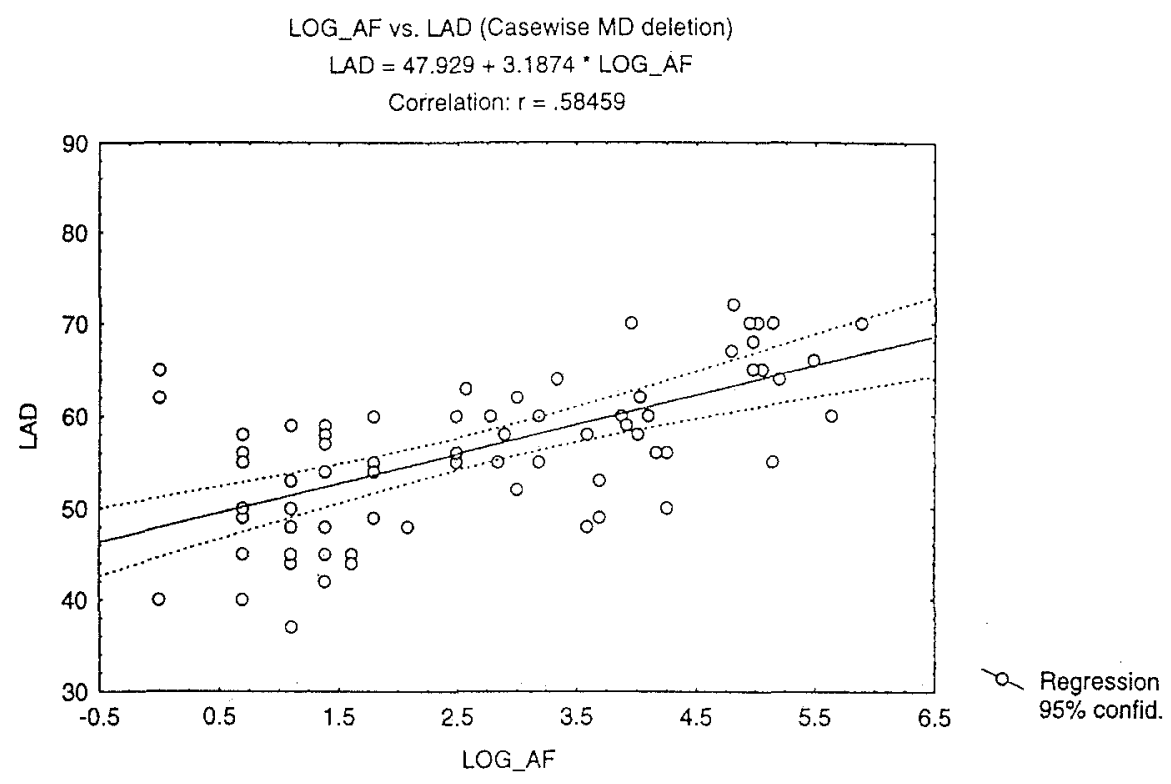

Fig. 3. Correlation between left atrial diameter $(L A D)$ and the logarithm of preoperative duration of $A F$.

of the patients who had a left atrial diameter greater than $60 \mathrm{~mm}$. As a consequence, precise prediction remains problematic even though, overall, the predictive value is satisfactory.

The low incidence of thromboembolic complications may be due in part to the limited follow-up of the series and to an insufficiently precise neurologic follow-up. Abnormal findings on computed tomographic scanning of the brain are prevalent in patients with chronic $\mathrm{AF}$, indicating prior silent cerebral infarction, even in the absence of clinically evident stroke. ${ }^{11}$

With regard to secondary conversion, the presence of preoperative $\mathrm{AF}$ has no predictive value. Although the return to stable postoperative SR may yield a different prognosis, a significant difference was not evident in the present series (see Figs. 1 and 2). In addition to this influence on survival, the functional improvement related to SR also needs to be taken into account. ${ }^{12}$

The indications for mitral valve repair should take these factors into account and optimize the probability of postoperative restoration of SR. The simplest solution would be to operate earlier to increase the proportion of patients who have SR at the time of the operation. This proportion is, in fact, very dependent on patient recruitment. Depending on the series, $30 \%$ to $60 \%$ of patients already have AF when they undergo surgery. ${ }^{13,14}$ This proportion is probably lower for teams with extensive and longstanding experience in this surgical field. Cardiolo- gists in those circumstances have greater awareness of, and confidence in, the results of valve repair. ${ }^{15-17}$ AF development must be considered a decisive turning point in the course of mitral regurgitation, even though it may be problematic to propose surgical therapy to patients who have few or no symptoms. However, this approach is encouraged by the well-established long-term quality of the results ${ }^{18}$ of mitral valve repair in patients operated on with NYHA class I or II disease.

Once AF has become established, surgical methods of treating it may be considered, such as the Cox maze procedure, the efficacy of which has been firmly established. ${ }^{19}$ However, the Cox maze procedure complicates mitral repair and significantly increases crossclamp time. ${ }^{20}$ Consequently, the indications must be carefully evaluated and the procedure restricted to patients with a low probability of returning to SR. On the basis of our results, the Cox maze procedure could be used in cases of AF lasting longer than 1 year or when the left atrial diameter is greater than $60 \mathrm{~mm}$. These considerations nonetheless have to be counterbalanced by the fact that the patient population concerned is in a highly precarious clinical and hemodynamic state, arguing against complex surgery. If the current series is analyzed including only patients who had AF for more than 1 year, a left atrial diameter greater than $60 \mathrm{~mm}$, an ultrasonic ejection fraction greater than $55 \%$, and only requiring simple valve 
repair (isolated posterior leaflet prolapse), nine patients could have benefited from a Cox maze procedure, that is, a quarter of the patients who had $\mathrm{AF}$ for more than 1 year and $4.7 \%$ of the whole series. The proportion could be increased provided that the Cox maze procedure does not result in prohibitive excess morbidity.

\section{Conclusion}

Postoperative recovery of SR after mitral valve repair is a positive prognostic factor. This objective strongly suggests prompt surgical treatment of patients with SR or with recent-onset chronic AF (less than 1 year) so as to optimize the probability of success. Surgical treatment of AF may be appropriate if restricted to patients with a long-standing history of AF or with a left atrial diameter greater than $60 \mathrm{~mm}$ and whose left ventricular function has been relatively well preserved.

\section{REFERENCES}

1. David TE, Armstrong S, Sun Z. Left ventricular function after mitral valve surgery. J Heart Valve Dis 1995;4:S17580.

2. Pitarys CJ, Forman MB, Panayiotou H, Hansen DE. Longterm effects of excision of the mitral apparatus on global and regional ventricular function in humans. J Am Coll Cardiol 1990;15:557-63.

3. Cox JL, Schuessler RB, D'Agostino HR, Stone CM, Chang $\mathrm{BC}$, Cain ME, et al. The surgical treatment of atrial fibrillation. III. Development of a defnitive surgical procedure. J Thorac Cardiovase Surg 1991;101:569-83.

4. Edmunds LH, Clark RE, Cohn LH, Weisel RD. Guidelines for reporting morbidity and mortality after cardiac valvular operations. J Thorac Cardiovasc Surg 1988;96:351-3.

5. Guidelines for statistical reporting in articles for medical journals. Ann Intern Med 1988;108:226-73.

6. Kannel WB, Abbott RD, Savage DD, McNamara PM. Epidemiologic features of chronic atrial fibrillation: the Framingham study. N Engl J Med 1982;306:1018-22.

7. Wolf PA, Dawber TR, Thomas HE Jr, Kannel WB. Epidemiologic assessment of chronic atrial fibrillation and risk of stroke: the Framingham study. Neurology 1978;28: 973-7.

8. Gosselink AT, Crijns HJ, Hamer HPM, Hillege H, Lie Ki. Changes in left and right atrial size after cardioversion of atrial fibrillation: role of mitral valve disease. J Am Coll Cardiol 1933;22:1666-72.

9. Manning WJ, Di Silverman, Katz SE, Riley MF, Come PC, Doherty RM, et al. Impaired left atrial mechanical function after cardioversion: relation to the duration of atrial fibrillation. J Am Coll Cardiol 1994;23:1535-40.

10. Sanfilippo AJ, Abascal VM, Sheehan M, Oertel LB, Harrigan $\mathrm{P}$, Hugues RA, et al. Atrial enlargement as a consequence of atrial fibrillation: a prospective echocardiographic study. Circulation 1990;82:792-7.

11. Petersen P, Madsen EB, Brun B, Pedersen F, Gyldensted C, Boysen $G$. Silent cerebral infarction in chronic atrial fibrillation. Stroke 1987;18:1098-100.

12. Triposkiadis F, Trikas A, Tentolouris K. Effect of atrial fibrillation on exercise capacity in mitral stenosis. Am J Cardiol 1995;76:282-6.

13. Brodell GK, Cosgrove D, Schiavone W, Underwood DA, Loop FD. Cardiac rhythm and conduction disturbances in patients undergoing mitral valve surgery. Cleve Clin J Med 1991;58:397-9.

14. Chua LY, Schaff HV, Orszulak TA, Morris JJ. Outcome of mitral valve repair in patients with preoperative atrial fibrillation. J Thorac Cardiovasc Surg 1994;107:408-15.

15. Alvarez JM, Deal CW, Loveridge K, Brennan P, Eisenberg $\mathrm{R}$, Ward $\mathrm{M}$, et al. Repairing the degenerative mitral valve: ten- to fifteen-year follow-up. J Thorac Cardiovasc Surg 1996;112:238-47.

16. Carpentier A. Cardiac valve surgery: the "French correction." J Thorac Cardiovasc Surg 1983;86:323-37.

17. Orszulak TA, Schaff HV, Danielson GK, Piehler JM, Pluth JR, Frye RL, et al. Mitral regurgitation due to ruptured chordae tendineae: early and late results of valve repair. J Thorac Cardiovasc Surg 1985;89:491-8.

18. Loop FD. Long-term results of mitral valve repair. Semin Thorac Cardiovasc Surg 1989;1:203-10.

19. Cox JL, Schuessler RB, Boineau JP. The surgical treatment of atrial fibrillation: summary of the current concepts of mechanisms of atrial flutter and atrial fibrillation. J Thorac Cardiovasc Surg 1991;101:402-5.

20. Hioki M, Ikeshita M, Iedokoro $Y$, Nitta $T$, Harada $A$, Asana $\mathrm{T}$, et al. Successful combined operation for mitral stenosis and atrial fibrillation. Ann Thorac Surg 1993;55:776-8. 